\title{
Channel Management für Krankenhäuser - Social Media im Klinik-Marketing
}

\author{
Christian Stoffers
}

(C) Springer-Verlag Wien 2012

Das Internet hat die Entwicklung der Wirtschaft in den letzten zwei Jahrzehnten maßgeblich beeinflusst. Kaum ein anderes Medium zuvor vermochte in so kurzer Zeit eine derartige Verbreitung zu erlangen. Obgleich offen ist, wie sich das Internet entwickelt, wie neue Technologien das Nutzerverhalten beeinflussen und ob Social Media letztendlich zu einer weiteren Revolution in der Kommunikation eines Krankenhauses führen werden, kann ein großes Potenzial insbesondere für Unternehmen der Gesundheitswirtschaft konstatiert werden. Dieses wird umso deutlicher, je intensiver man Unternehmen und Märkte analysiert: Wurden beispielsweise im St. Marien-Krankenhaus Siegen früher primär Patienten stationär behandelt, so werden heute neben 20.000 stationären fast 70.000 ambulante Fälle versorgt. Gleichzeitig entwickelte sich das Unternehmen mit über 1.700 Mitarbeitern zu einem der größten Arbeitgeber der Region Siegen-Wittgenstein. Das Unternehmen, das vor zehn Jahren noch über keine Website verfügte, befindet sich demnach in einem massiven Wandel, wobei sich dieser auch in der Kommunikation und den eingesetzten Medien widerspiegelt.

Social Media eröffnen dem St. Marien-Krankenhaus die Möglichkeit, auf völlig neue Weise mit seinen Austauschpartnern zu kommunizieren. Denn die klassischen Instrumente der Kommunikationspolitik bieten im Gegensatz zu den Social Media keine unmittelbaren oder nur verzögerte Reaktionsmöglichkeiten für den Empfänger der Botschaft.
Social Media lassen sich somit als sinnvolle Ergänzung der Kommunikation kennzeichnen. Dem St. Marien-Krankenhaus ist es mit einem komplexen Channel Management, dessen Grundlage qualitative und quantitative Kennzahlen sind, gelungen, mit unterschiedlichen Medien das klassische Sender-Empfänger Modell zu überwinden und dabei in direkten Kontakt mit seinen Austauschpartnern zu treten. Dabei werden wöchentlich bis zu 30.000 unterschiedliche Personen mittels interaktiven Dialogs zwischen Krankenhaus und seinem Austauschpartner erreicht. Analyse-Tools helfen hier dem Krankenhaus, geeignete Ansatzpunkte zu finden und bilden somit die methodische Grundlage.

Die Austauschpartner werden in Social Media ohne großen zeitlichen oder finanziellen Aufwand zum Sender von Informationen für das St. Marien-Krankenhaus. Sie können ihre Meinungen äußern, von Problemen berichten, Vorschläge machen und so zu einer Informationsquelle von großem Wert für das Krankenhaus werden. Auch können sie hierüber im Sinne eines viralen Marketing-Ansatzes eingebunden werden, wie es unterschiedliche Kampagnen des St. Marien-Krankenhauses in Social Media zeigen. Dies alles hat dazu geführt, dass das St. Marien-Krankenhaus - ermittelt in einer Imageanalyse - aus dem Channel Management einen messbaren Vorteil in der öffentlichen Wahrnehmung gewonnen hat. 